\title{
ChemComm
}

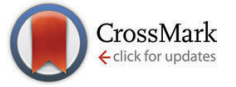

Cite this: Chem. Commun., 2014, 50, 13636

\section{Highlights from Faraday Discussion 168: Astrochemistry of Dust, Ice and Gas, Leiden, The Netherlands, April 2014}

\author{
John D. Thrower, ${ }^{\star a}$ Sergio loppolo ${ }^{\text {bc }}$ and Catherine Walsh ${ }^{d}$
}

DOI: $10.1039 / \mathrm{c} 4 \mathrm{cc} 90397 \mathrm{~h}$

www.rsc.org/chemcomm

The morning of the 7th April 2014 saw the arrival of around 120 astrochemists from around the globe in the quiet South Holland town of Noordwijkerhout, just outside Leiden. The delegates for Faraday Discussion 168 were treated to a premier view of Holland's famous tulip fields en route via coach from Schiphol airport (Fig. 1). Hosted at the $\mathrm{NH}$ Conference Centre Leeuwenhorst, FD168 was the 5th Faraday Discussion in the field of astrochemistry, following from previous discussions on the Chemistry of the Interstellar Medium in 1992, Chemistry and Physics of Molecules and Grains in Space in 1998, Chemical Evolution of the Universe in 2005 and the Chemistry of Planets in 2010. Faraday Discussions have a legacy going back 100 years and have covered a wide range of topics in physical chemistry. The field of astrochemistry is an excellent example of an interdisciplinary field: it brings together astronomers, chemists, and physicists working on observations, theory, and experiments to advance our

\footnotetext{
${ }^{a}$ Physikalisches Institut, Westfälische Wilhelms-Universität, D-48149 Münster, Germany. E-mail:john.thrower@uni-muenster.de

${ }^{b}$ Division of Geological and Planetary Sciences, California Institute of Technology, 1200 E. California Blvd., Pasadena, USA ${ }^{c}$ Institute of Molecules and Materials, Radboud University Nijmegen, P.O. Box 9010, 6500 GL Nijmegen, The Netherlands

${ }^{d}$ Leiden Observatory, Leiden University, P.O. Box 9513, 2300 RA Leiden, The Netherlands
}

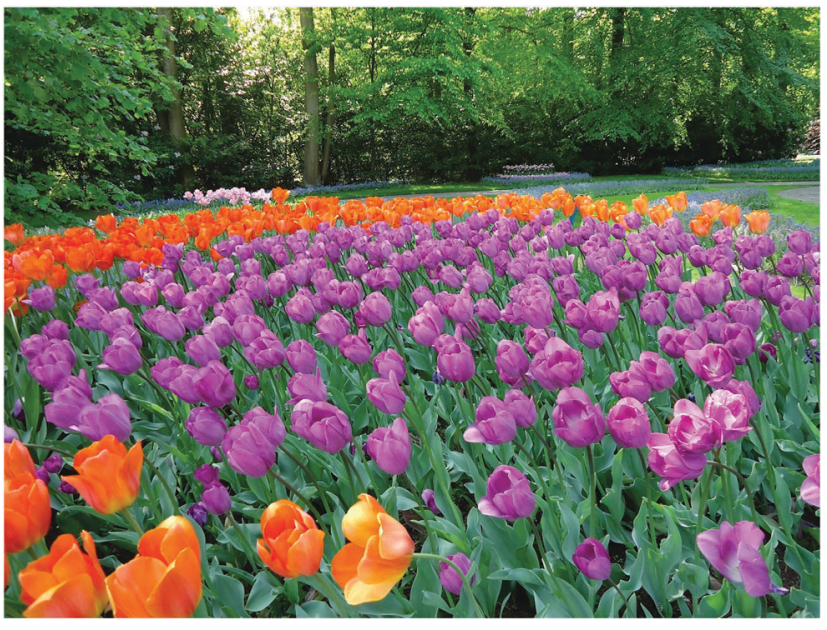

Fig. 1 FD168 delegates were greeted with views of the famous Dutch bulb fields (Credit: S. Ioppolo).

understanding of the unique and exotic chemistry that occurs in space .

The weather on arrival was better than expected for this typically wet, yet lush, region of the Netherlands. Upon arrival, attendees were efficiently registered and presented with their conference materials, including a small taste of the Netherlands in the form of a pack of stroopwafels. Scientific discussions began in earnest over a buffet lunch adjacent to the main auditorium, before the official opening and welcome by Martin McCoustra (HeriotWatt University, Edinburgh), the chair of the scientific committee comprising Wendy Brown (University of Sussex), Robin Garrod (Cornell University), Harold Linnartz (Leiden University), Anthony Meijer
(University of Sheffield), and Tom Millar (Queen's University Belfast). In addition to introducing the 10 invited speakers, he outlined the unique form of Faraday Discussion meetings, which is somewhat different to the majority of scientific meetings. Speakers are asked to prepare a paper on their research several months prior to the meeting. These are then peer-reviewed and the preprints made available to the delegates several weeks ahead of the discussion, allowing for pre-digestion of the material. Speakers have a strictly enforced - using the famous Faraday traffic lights - 5 minute slot to present the key points of each paper, with extended discussions dominating the programme. Importantly, the discussions 
are recorded and form an integral part of the published discussion volume, becoming citeable literature in their own right.

A 50 minute introductory lecture on the general topic of astrochemistry was given by Ewine van Dishoeck (Leiden University). This comprehensive lecture helped to 'set the scene' for the remainder of the meeting and highlighted recent observational advances, including results from the Atacama Large Millimeter/ Submillimeter Array (ALMA) and the Herschel Space Observatory. Advances in theoretical and laboratory astrochemistry are very much driven by new observations from state-of-the-art telescopes.

The opening session on "Observations on dust, ice and gas relevant to astrochemistry" was chaired by Tom Millar (Queens University Belfast). Klaus Pontoppidan (Space Telescope Science Institute, Baltimore) kicked off the presentations by describing a new method for deriving molecular abundances through observations of molecular emission lines from protoplanetary disks. Protoplanetary disks are remnant tori of dust and gas around young stars within which planets form. The 'planet-forming' region of disks is best probed via mid-infrared observations using space-based facilities such as the Spitzer Space Telescope. Traditionally, these observations are analysed using a simple slab model, assuming a single temperature and density. In reality, disks have strong temperature and density gradients which can influence the molecular line emission. Pontoppidan and Blevins ${ }^{1}$ propose a more complex 'retrieval' method in which a large parameter space is investigated in parallel with sophisticated radiative transfer. Their preliminary results and comparison with Spitzer data show the model's potential to constrain the molecular concentration and distribution within the planet-forming zone.

The following paper was presented by Karin Öberg (Harvard University). Öberg et $a .^{2}$ present new Submillimeter Array (SMA) observations of line emission from complex organic molecules (COMs) towards several massive young stellar objects (MYSOs). MYSOs are objects which will evolve to form massive stars or clusters of stars. They typically have very complex spectra with a high density of spectral lines,

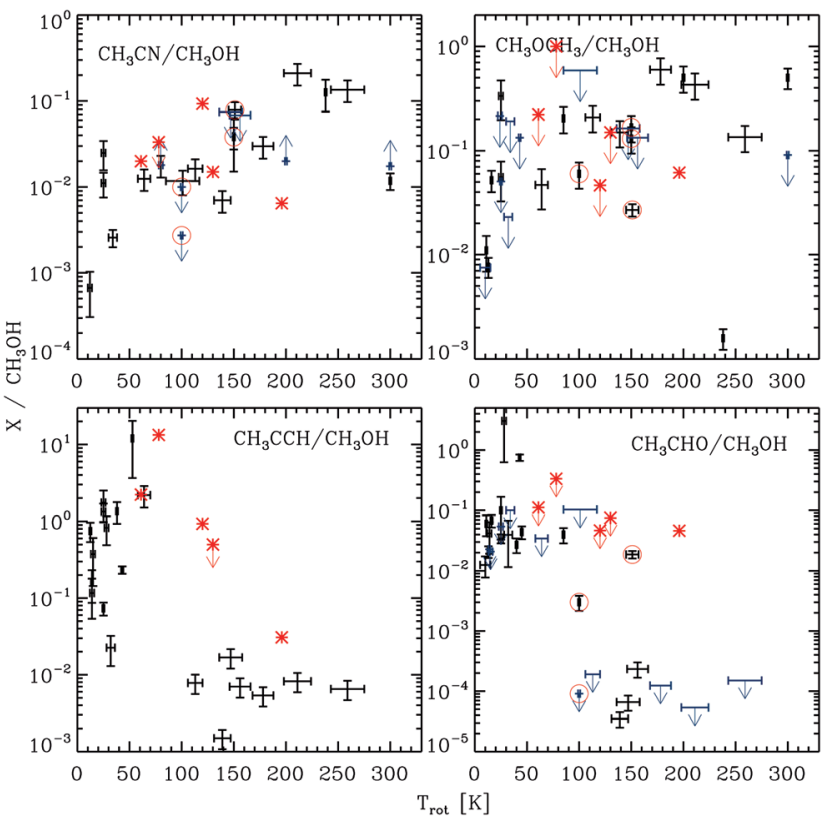

Fig. 2 Öberg et al. compare column density ratios of various species for a range of sources in the literature (black) and several Massive Young Stellar Objects (MYSOs; red). The observed trends indicate that complex organic formation is triggered in MYSOs as they heat up. ${ }^{2}$

with contributions from many complex molecules. The formation of complex molecules in interstellar and circumstellar environments is a current 'hot topic' in astrochemistry, and is thought to occur on and within ice mantles on the surfaces of dust grains. Öberg et al. mapped the molecular line emission across several sources from COMs including propyne $\left(\mathrm{CH}_{3} \mathrm{CCH}\right)$, acetonitrile $\left(\mathrm{CH}_{3} \mathrm{CN}\right)$ and acetaldehyde $\left(\mathrm{CH}_{3} \mathrm{CHO}\right)$ with the goal of looking for trends in molecular excitation and distribution with temperature (see Fig. 2). $\mathrm{COMs}$ such as $\mathrm{CH}_{3} \mathrm{CCH}$ and $\mathrm{CH}_{3} \mathrm{CHO}$ can form at low temperatures with $\mathrm{CH}_{3} \mathrm{CHO}$ also having a formation route at higher temperatures. The observations support temperature-activated chemistry as a route to several complex organic molecules allowing their use as a potential evolutionary tracer of MYSOs.

Viviana Guzmán (Institut de Radioastronomie Millimétrique - IRAM) subsequently presented some exciting detections of COMs in a photondominated region (PDR) for the first time. $^{3}$ The observed species include formic acid $(\mathrm{HCOOH})$, ketene $\left(\mathrm{CH}_{2} \mathrm{CO}\right)$, acetaldehyde $\left(\mathrm{CH}_{3} \mathrm{CHO}\right)$ and propyne $\left(\mathrm{CH}_{3} \mathrm{CCH}\right)$. PDRs form the interface between dark dense molecular clouds and the irradiated interstellar medium, and as such are considered to be relatively harsh environments. That gas-phase COMs are able to form and survive in PDRs is of astrochemical significance because it suggests that radiation processing of ice mantles is important for building chemical complexity, verifying a result that has been known from laboratory experiments for some decades.

In the closing paper of the day, Ted Bergin (University of Michigan) moved the discussion on to more 'local' matters by presenting a paper on the origin of carbon in terrestrial planets. Carbon is an element of extreme astrobiological significance and provides the 'backbone' for many of the molecules necessary for life. Bergin et $a l^{4}{ }^{4}$ discuss the various observational constraints on the carbon budget in star-forming regions and extrasolar systems in comparison with the known carbon content in the Solar System (including the Sun, the Earth, comets, and chondritic meteorites). They suggest that much of the carbon in the pre-solar nebula (the protoplanetary disk from which the Solar System formed) may have been in volatile form, explaining the lack of solid and refractory carbon in the current Solar System. However, observations of line emission from $\mathrm{C}^{18} \mathrm{O}$ and $\mathrm{HD}$ from our nearest early Solar System analogue, 
TW Hydrae, indicate that CO is not the main carbon reservoir in this particular disk. The authors present new models investigating mechanisms for the extraction of carbon from CO. They postulate that the destruction of gas-phase CO via reaction with $\mathrm{He}^{+}$ions creates $\mathrm{C}^{+}$ions, which are very reactive and lead to an increase in other carbon reservoirs, such as complex molecules. This can only occur where $\mathrm{CO}$ is not in ice form; hence the carbon budget in comets and other icy planetesimals is preserved.

The first day of the conference also hosted the first discussion session, leading to lively debates on the presented papers. Several comments and questions concerning the opening lecture by Ewine van Dishoeck arose with suggestions for new target species and reactions for the experimental and computational chemistry community, in advance of observational results from new facilities (e.g., ALMA and JWST). Prof. van Dishoeck also highlighted the need for "back to basics" models to study the role of key reactions upon which Prof. Meuwly commented on the now realistic use of sophisticated computational methods to study reaction kinetics of simple systems. Klaus Pontoppidan was the next to receive comments and questions. Several in the audience were interested in the low $\mathrm{CO}_{2}$ abundances derived using their protoplanetary disk observations. Prof. Kamp suggested including a vertical abundance profile in additional to a radial profile and also postulated that a significant molecular reservoir may be hidden by dust. There was much interest in Ted Bergin's paper with comments and discussion concerning the different carbon reservoirs in protoplanetary disks. In particular, Prof. Linnartz highlighted the importance of PAHs, both as a carbon reservoir and as a source of carbon for forming more simple molecules, the so-called "top-down" approach, a sentiment echoed by many others in the audience. The chemistry of COMs was also extensively discussed, both in relation to carbon budget $\left(\sim 10^{-5}\right.$ with respect to $\mathrm{H}_{2}$ as estimated by Karin Öberg) and potential formation mechanisms. Dr Ellinger was the first to comment on the importance of chemical (reactive) desorption given the exothermicity of grain-surface reactions, a subject that was to be revisited over the coming days. Prof. Millar commented on the use of isotopologues to constrain the different pathways to COM formation. On the comparison between models and observations, Karin Öberg stressed the need for larger observational sample sizes (sources and species) and Dr Garrod also commented on the need for well-constrained physical models for meaningful comparisons. The first detection of COMs in a PDR also prompted some discussion. Dr Cuppen and Dr Fayolle commented on the recent experimental results on methanol photodesorption which suggest that radicals and "daughter" species are preferentially ejected into the gas; however, Profs van Dishoeck and Fillion remarked on the importance of indirect desorption via either a "kick-out" mechanism or via electronic excitation of CO molecules. The first discussion, on the whole, highlighted many important scientific questions that the observational, modelling, and laboratory communities can realistically work towards answering in the coming years.

Immediately following the discussion session, delegates made their way to the poster session. The number of posters, at 73, was impressive, allowing almost every delegate to contribute. Many aspects of astrochemistry, including gas phase chemistry, observations, modelling, surface reactions and interstellar ices, were represented, and stimulating discussions continued over dinner.

Tuesday morning was devoted to the discussion of "Laboratory astrochemistry of dust and ice" introduced by the session chair Wendy Brown (University of Sussex). Laboratory work is a fervid branch of the field and the last few decades have witnessed a blooming of new and more sophisticated laboratory techniques which have allowed the investigation of interstellar ice formation under fully controlled conditions. However, to date, many of the surface routes linked to the formation of interstellar COMs have not yet been verified experimentally. Even simple processes such as diffusion rates of reactants on and within an ice remain largely unquantified. Emanuele Congiu (LERMA: Universities of Paris and Cergy-Pontoise) opened the discussion showing their latest results on the efficient diffusion of $\mathrm{O}$ atoms at low temperatures on water ice, silicates, and graphite. ${ }^{5}$ They report an unexpected correlation between ordered surfaces and efficient diffusion of $\mathrm{O}$ atoms. In all cases, the observed diffusion rates are larger than previously predicted, suggesting that $\mathrm{O}$ atoms can diffuse by quantum mechanical tunnelling at temperatures as low as $6.5 \mathrm{~K}$. The latter result has important implications in astrochemistry, since there is a point in the evolution of molecular clouds where $\mathrm{H}$ and $\mathrm{O}$ atoms have comparable abundances because of the efficient conversion of atomic $\mathrm{H}$ to molecular $\mathrm{H}_{2}$. If $\mathrm{O}$ atoms are mobile on grain surfaces at low temperatures, then oxygen addition reactions can potentially play an important role in the synthesis of large organic molecules.

Appropriately, the next paper was presented by Stephen Price (University College London) and considered the single and double addition of $\mathrm{O}$ atoms to propyne $\left(\mathrm{CH}_{3} \mathrm{CCH}\right)$ ice. ${ }^{6}$ The authors report the efficient formation of propenal $\left(\mathrm{CH}_{2} \mathrm{CHCHO}\right)$ at temperatures below $100 \mathrm{~K}$. Price et al. show that, as the surface temperature decreases, the reaction yield increases to reach a maximum at $50 \mathrm{~K}$ and then a minimum at $30 \mathrm{~K}$. Both the Langmuir-Hinshelwood (where both reactive species are initially adsorbed and thermalized on the surface) and the Eley-Rideal (where a thermalized surface molecule reacts with an incoming, and potentially energetic, species from the gas phase) mechanisms are considered in their model to explain the observed experimental trend. They derive an activation energy of $\sim 160 \mathrm{~K}$ for the reaction, $\mathrm{CH}_{3} \mathrm{CCH}+\mathrm{O}$. At surface temperatures below $30 \mathrm{~K}$, the yield of reaction rises again indicating an alternative (barrierless) reaction mechanism, active only at low temperatures. Finally, Price and colleagues also observe the formation of an additional surface product $\left(\mathrm{C}_{3} \mathrm{H}_{4} \mathrm{O}_{2}\right)$ corresponding to the barrierless addition of one $\mathrm{O}$ atom to propenal.

The third paper, presented by Yasuhiro Oba (Hokkaido University) described a detailed work on the reaction kinetics and isotope effect of water formation through the hydrogenation/deuteration of $\mathrm{H}_{2} \mathrm{O}_{2} / \mathrm{D}_{2} \mathrm{O}_{2}$ ice. ${ }^{7}$ Water is the most abundant species in interstellar ices and its 
formation proceeds through the hydrogenation of $\mathrm{O} / \mathrm{O}_{2} / \mathrm{O}_{3}$ at low temperatures. Hydrogen peroxide is an intermediate product of the hydrogenation of molecular oxygen ice. The authors formed a layer of pure $\mathrm{H}_{2} \mathrm{O}_{2}$ by co-depositing $\mathrm{O}_{2}$ and $\mathrm{H}$ at $45-50 \mathrm{~K}$. These experiments allowed them to compare the efficiency of the reactions $\mathrm{H}_{2} \mathrm{O}_{2}+\mathrm{H}$ and $\mathrm{H}_{2} \mathrm{O}_{2}+\mathrm{D}$ at $10-30 \mathrm{~K}$. Despite the large activation barriers $(\sim 2000 \mathrm{~K})$, both reactions proceed at low temperatures with the reaction rate for $\mathrm{H}$ atoms determined to be 45 times faster than that for D atoms at $15 \mathrm{~K}$. This large isotope effect indicates that the reactions proceed via quantum mechanical tunnelling.

Markus Meuwly (University of Basel) introduced the final paper of the morning session which provided a complementary theoretical discussion concerning the diffusion of oxygen atoms during water formation in amorphous interstellar ices. ${ }^{8}$ Their work supports the experimental results of Congiu and co-authors on the diffusion of $\mathrm{O}$ atoms at low temperatures. The authors present classical molecular dynamics (MD) simulations of O-atom migration in amorphous water ice that use physically motivated force fields. Their calculations show that hopping of $\mathrm{O}$ atoms occurs on the sub-nanosecond time scale at temperatures below $200 \mathrm{~K}$ (see Fig. 3) and yield estimates for diffusion rates of $\sim 5$ to $15 \mathrm{~ns}^{-1}$ and barrier heights of $\sim 500 \mathrm{~K}$ or less. Therefore, this work further supports the hypothesis that oxygendriven chemistry within ice mantles on the surfaces of dust grains may play a more important role in interstellar environments than previously assumed.

After a short break, the subject of atomgrain interactions was extended to higher temperature environments (e.g. photon dominated regions; PDRs) where atoms can form chemical bonds with, for example, carbonaceous grains and molecules such as polycylic aromatic hydrocarbons (PAHs). Liv Hornekær (Aarhus University) described her group's recent experimental studies of $\mathrm{H}$-atom addition to PAH molecules. ${ }^{9}$ PAHs are thought to account for more than $10 \%$ of the carbon budget and are responsible for infrared emission features observed along many lines of sight in the ISM. Through thermal desorption and mass spectrometric measurements, Skov et al. show evidence for the formation of the fully hydrogenated state of coronene upon $\mathrm{H}$-atom addition to $\mathrm{C}_{24} \mathrm{H}_{12}$ and for abstraction reactions that lead to $\mathrm{H}_{2}$ formation. The authors also discuss a series of kinetic simulations aimed at extracting reaction cross-sections for both addition and abstraction reactions.

Nigel Mason (Open University, Milton Keynes) discussed the important role played

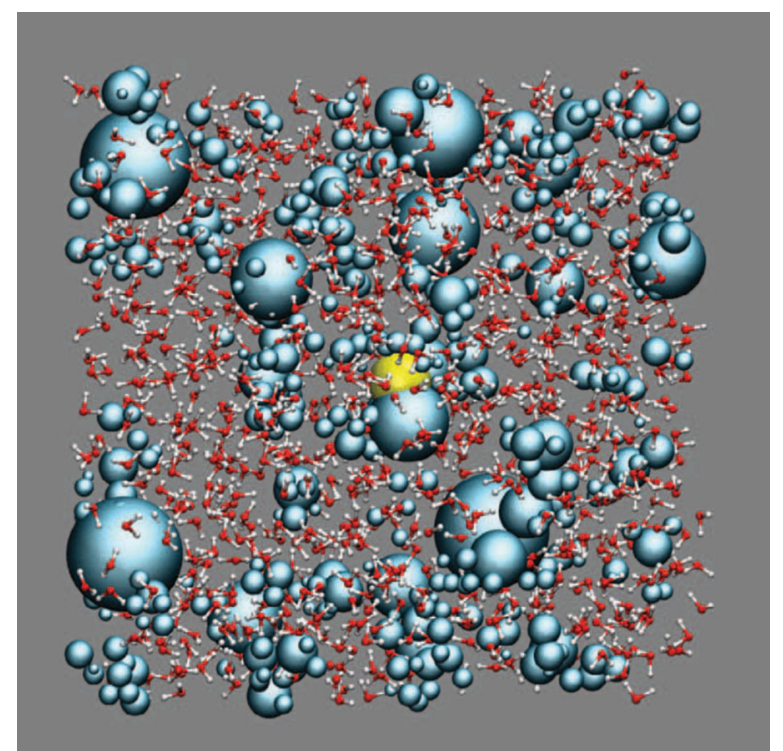

Fig. 3 Lee et al. use molecular dynamics (MD) simulations to investigate the mobility of oxygen atoms in amorphous water as a function of temperature. ${ }^{8}$ by secondary electrons, generated by the passage of primary cosmic ray particles through interstellar ices, in initiating chemical transformations within the ices. ${ }^{10}$ Experiments in which model interstellar ices are exposed to a beam of low energy electrons can help to elucidate the reaction pathways initiated by this cascade. In the paper by Mason et al., the irradiation of methanol and methanol-ammonia mixed ices is used to exemplify this rich chemistry. Formed though the hydrogenation of $\mathrm{CO}, \mathrm{CH}_{3} \mathrm{OH}$ is a key carbon containing component of interstellar ices and often considered the starting point for the formation of more complex organic species. $\mathrm{CO}$ and $\mathrm{CO}_{2}$ are observed to be formed during electron irradiation of methanol, while $\mathrm{NH}_{2}$ radicals are generated from ammonia. Reactions between reactive species formed in this way can then go on to form more complex species. Also of interest was the discussion of the dissociative electron attachment (DEA) mechanism through which reactive intermediates can form efficiently at very low electron energies, even below the dissociation energy of the molecule.

Continuing on this theme, Christopher Arumainayagam (Wellesley College) reported on a mass spectrometric study of the reaction products formed through the low energy electron irradiation of condensed methanol $\left(\mathrm{CH}_{3} \mathrm{OH}\right){ }^{11}$ In these elegant experiments, his group heated low energy electron irradiated methanol ices, detecting desorbing reaction products using mass spectrometry; this revealed the presence of eleven distinct products. These range in complexity from formaldehyde $\left(\mathrm{H}_{2} \mathrm{CO}\right)$ to 1,2,3-propanetriol $\left(\mathrm{HOCH}_{2} \mathrm{CHOHCH}_{2} \mathrm{OH}\right)$. The richness of the chemistry derived from this relatively simple species highlights the importance of detailed laboratory measurements in unravelling the complexities of radiation driven ice processing.

To close the session, Belén Maté (Instituto de Estructura de la Materia) compared low energy electron and UV photon irradiation for two specific cases: (a) the dehydrogenation of hydrogenated amorphous carbon (HAC) dust surfaces, and (b) the destruction of glycine. ${ }^{12}$ It remains unclear whether prebiotic species potentially formed in the ISM, 
such as simple amino acids, survive for a sufficiently long time to become incorporated into planetesimals in a forming planetary system. By performing infrared spectroscopy on the irradiated samples, Maté et al. conclude that HAC is relatively stable to both forms of processing whilst glycine is destroyed far more efficiently. They find that electron irradiation dominates in the outer layers of the ice sample as a result of the penetration depth, sounding a note of caution on the care needed when using several keV electrons to simulate processes induced by the passage of cosmic ray particles through interstellar ices.

In the subsequent discussion, the role of quantum tunnelling in atomic oxygen diffusion was discussed at length. Prof. Meuwly noted that departure from Arrhenius behaviour is not necessarily sufficient to infer that tunnelling is involved, especially when a distribution of diffusion barriers is present. Dr Congiu confirmed that the investigation of isotope effects is indeed necessary to confirm that tunnelling is at play. When Dr Garrod commented on the fact that Eley-Rideal and hot-atom processes cannot be ruledout a priori, Dr Congiu explained that they actually implemented those mechanisms in their model. However, the outcome of their study indicates that quantum tunneling diffusion becomes dominant. Dr Hama highlighted the importance of determining correct surface diffusion rates, and indicated that the temperature dependence of O-atom diffusion rate observed in Dr Congiu's experiments could be explained by thermal hopping without tunnelling diffusion. Dr Ioppolo and Prof. McCoustra pointed out that appropriate laboratory techniques for controlling the degree of porosity and crystallinity of $\mathrm{H}_{2} \mathrm{O}$ films are needed to study mechanisms such as the diffusion of species on different surfaces. Prof. Herbst suggested to Prof. Price and co-workers to look into the sub-monolayer regime to better understand absolute rate coefficients of surface reactions and the mechanisms at play with the goal of extending laboratory results to the interstellar medium. The same regime was used by Dr Oba to study water formation from a pure thin layer of $\mathrm{H}_{2} \mathrm{O}_{2}$. In this case, the authors studied the water-formation reaction-scheme starting from one of the products of $\mathrm{O}_{2}$ hydrogenation. Although this strategy narrows the reaction pathway, a multiple formation-path for water ice cannot be excluded, as pointedout by Dr Ioppolo. In the discussion of electron-induced processes Prof. Rawlings indicated that experimentalists report cross-sections and yields, while modellers wish to incorporate energy integrated rate constants in their models. This would require knowledge of the effective flux of secondary electrons, something that is highly reliant on Monte Carlo simulations, as pointed out by Prof. Arumainayagam. Dr Woods and Prof. Kamp questioned the extent to which photon and electron driven processes drive the formation of COMS and the reliability of extrapolating laboratory measurements to low flux interstellar environments. Prof. Mason explained that although many species can be formed through thermal routes, experiments have shown that non-thermal processes are equally as effective in forming COMs. He acknowledged that the timescale limitations of experiments make appropriate modelling essential while Prof. McCoustra highlighted the flux dependent measurements performed by experimentalists to ensure that only single-photon/particle excitation driven processes are probed. Prof. Linnartz added that hydrogenation reactions are probably the most efficient way to form simple hydrogenated species, but the relative efficiency of electron and photon induced processes in the formation of more complex molecules remains unclear. Prof. Arumainayagam and Prof. Mason agreed that this is an important point, although direct comparison between experiments using photon and electron irradiation remains difficult.

The first session after lunch, chaired by Robin Garrod (Cornell University), covered astrophysical modelling. Modelling is often required to both guide and interpret astrophysical observations. Anthony Jones (Institut d'Astrophysique Spatiale, France) returned to the theme of carbon, by presenting a paper discussing the "cycling of carbon into and out of dust" in the transition regions between the diffuse ISM and molecular clouds. ${ }^{13}$ In the ISM, carbon is present not only in ionic and molecular form, e.g., $\mathrm{C}^{+}, \mathrm{C}$, and $\mathrm{CO}$, but also in refractory form. These, sometimes nanoscale, particles are composed of small aliphatic and aromatic hydrocarbons and can undergo chemical processing by UV radiation. Jones et al. discuss and model the different production and destruction mechanisms for carbon-rich dust across the conditions found in the ISM. Their results suggest that in low extinction environments, small carbonaceous grains may consist of aromatic-rich carbon materials whereas in higher extinction regions, the carbonaceous material is more aliphatic in nature, leading to a variation in the optical properties of dust grains across the ISM.

The next paper changed tack, bringing the discussion back to ice formation and chemistry. Herma Cuppen (Radboud University Nijmegan) presented results from a microscopic kinetic Monte Carlo model developed to investigate the formation of water on interstellar grains via grain-surface reactions. Water, in both ice and gas form, is prevalent throughout many astrophysical environments. ${ }^{14}$ It is now generally understood that water forms primarily on grain surfaces; however, the exact mechanism for its formation and its chemistry remains an active area of research. Lamberts et al. explore the role of reaction exothermicity on the formation of water and related species, such as, $\mathrm{OH}$, $\mathrm{O}_{2} \mathrm{H}$, and $\mathrm{H}_{2} \mathrm{O}_{2}$. They find that allowing reaction products to convert their excess energy into translation motion or desorption helps to build a more compact ice. For the exothermic reaction $\mathrm{H}+\mathrm{O}_{2} \mathrm{H} \rightarrow \mathrm{OH}+\mathrm{OH}$, translational motion of the two products can help explain the detection of $\mathrm{OH}$ in laboratory experiments and corresponding lack of $\mathrm{H}_{2} \mathrm{O}_{2}$.

Appropriately, the next paper was presented by Bérengère Parise (University of Cardiff) on a deep search for gas-phase $\mathrm{H}_{2} \mathrm{O}_{2}$ in numerous and diverse astrophysical environments using the Atacama Pathfinder Experiment (APEX) telescope. ${ }^{15}$ The motivation was the first detection of gas-phase $\mathrm{H}_{2} \mathrm{O}_{2}$ in space, towards the cloud core, $\rho$ Oph A. $\mathrm{H}_{2} \mathrm{O}_{2}$ is a molecule of interest given its role in water chemistry. Parise et al. report no additional detections of $\mathrm{H}_{2} \mathrm{O}_{2}$ toward any of their sources with a typical upper limit to the abundance of $\leq 10^{-11}$ with respect to $\mathrm{H}_{2}$. Their chemical 
model shows that the production of gasphase $\mathrm{H}_{2} \mathrm{O}_{2}$ is very sensitive to temperature and reaches an appreciable abundance only in a narrow range between 20 and $30 \mathrm{~K}$. They suggest that $\rho \mathrm{Oph} \mathrm{A}$ is a special source in which the bulk of the material lies within this temperature range. That this source has a higher temperature may be related to its external environment: it is externally irradiated by nearby massive stars. Parise et al. suggest observations of additional externally heated sources may help prove this hypothesis.

The next paper, presented by Jonathan Rawlings (University College London), discussed a new mechanism for the production of gas phase COMs at low temperatures. ${ }^{16}$ It is believed that COMs are formed on or within ice mantles on dust grains. However, the recent detection of COMs in low temperature $(10 \mathrm{~K})$ environments has prompted many to revisit this mechanism. Rawlings et al. propose that gas phase COMs are formed via three-body gas-phase reactions following complete sudden sublimation of the ice mantle following an 'explosion' event triggered by the recombination of trapped hydrogen and radicals. They use this paradigm to model the formation of $\mathrm{C}_{2} \mathrm{H}_{4} \mathrm{O}_{2}$ isomers (methyl formate, glycolaldehyde, and acetic acid) as well as the simplest amino acid, glycine $\left(\mathrm{CH}_{2} \mathrm{NH}_{2} \mathrm{COOH}\right)$. Their model can explain the observed abundances of the $\mathrm{C}_{2} \mathrm{H}_{4} \mathrm{O}_{2}$ isomers provided the necessary fraction of precursor radicals are released from ice. They also predict a maximum fractional abundance of $\sim 10^{-10}$ relative to $\mathrm{H}_{2}$ for gas-phase glycine, in line with the constrained upper limits.

The final paper was presented by Catherine Walsh (Leiden University) and continued the theme of COM formation, this time, in protoplanetary disks. ${ }^{17}$ Walsh et al. present calculations of the abundance and distribution of COMs along streamlines in both isolated and externally irradiated protoplanetary disks. This latter aspect is important because the Sun may not have been born in isolation. Walsh et al. find that chemical complexity can increase along the accretion flow in isolated protoplanetary disks if only simple ices are injected. For the irradiated disk, COMs are unable to form via thermal grain-surface chemistry; hence the initial composition of the ice mantle is critical. COMs more complex than methanol do not survive transport through the disk due to thermal and radiation processing, indicating that dynamical timescales are very important. Also, complex molecules present in the planet-forming zone may have an interstellar origin provided the species are injected with a sufficiently high abundance. Planetesimals in irradiated disks may be composed of more simple ices, given the degree of thermal and radiation processing en route to the planet-forming zone.

The final discussion session of the day opened with a debate on the formation of dust in the ISM. Among the questions raised by Robin Garrod was the role of $\mathrm{O}$ and $\mathrm{N}$ atoms in carbon-dust formation which, according to Anthony Jones, can aid dust production and produce species which may help resolve the origin of DIBs (diffuse interstellar bands). The proposed model also revises current estimates for dust masses in the ISM downwards (prompted by a question from Prof. van der Tak). A topic highlighted for the future, was the role of charged grains on dust formation in the ISM (Prof. Daan Schram). The discussion moved on to consider the specific parameters adopted in the kinetic Monte Carlo models presented by Herma Cuppen, which allowed the authors to clarify the setup and methods adopted. The simulations support the non-detection of gas-phase $\mathrm{H}_{2} \mathrm{O}_{2}$ in all but a single astrophysical environment as presented in the paper by Bérengère Parise. Points raised for consideration on this latter issue included a measured rate coefficient for the reaction of $\mathrm{H}_{2} \mathrm{O}_{2}$ with $\mathrm{OH}$ (Ms Caravan) which may have an appreciable rate coefficient at low temperatures, and the use of isotopologues, e.g., HOOD (Prof. Linnartz). COM formation via gas-phase recombination reactions following the explosive release of grain mantle material through radical recombination reactions by Jonathon Rawlings generated a lively debate. Several audience members had reservations highlighting factors such as $\mathrm{H}$-atom concentration within the ice (Robin Garrod), survival of radicals following explosion (Eric Herbst), and the long lifetimes of molecular clouds compared with laboratory timescales
(Mr Fedoseev). Much of the debate surrounded the observable signature of such a mechanism. Proposed methods included using the relative abundances of structural isomers, e.g., $\mathrm{C}_{2} \mathrm{H}_{4} \mathrm{O}_{2}$ (Dr Ellinger), or targeted searches from COMs in $\mathrm{CO}$ icepoor regions, because the mechanism for methanol production is related to $\mathrm{CH}_{4}$ and not CO (Jonathon Rawlings). The final debate centred on COM formation in protoplanetary disks where the importance of dynamics on the chemistry was highlighted (Dr Semenov), as was the importance of a complete treatment of bulk ice chemistry as well as surface chemistry (Prof. Kaiser), and the use of sensitivity analyses from grain surface chemistry to identify critical parameters (Dr Heays). A potential topic for the future was suggested by Anthony Jones and concerned the influence of the presence of a hydrocarbon-rich material at the ice-grain interface in protoplanetary disks which Catherine Walsh agreed may help build chemical complexity in disks. This discussion nicely highlighted the need for ongoing dialogue between the modelling and laboratory communities.

After the conclusion of the afternoon session, a short break was provided in the programme before the attendees were invited to begin the evening's festivities with pre-dinner drinks. Places were then taken for the three course conference dinner, after which Andrew Mount (University of Edinburgh), chair of the Faraday Standing Committee on Conferences, spoke on the history of the Faraday Discussion series and introduced the tradition of the Faraday Loving Cup, which then made its way around the room. FD168 also saw the joint award of the Skinner Prize for best student poster to Rebecca Caravan (University of Leeds) for her poster describing "Chemical mechanisms operating at very low temperatures to enhance the rates of gas phase reactions relevant to interstellar environments", and Robert Frigge (University of Münster) for his poster on "Femtosecond laserinduced desorption of atomic and molecular hydrogen isotopes from graphite".

The final session, on Wednesday morning, was chaired by Harold Linnartz (Leiden University) and centred on "New directions in solid and surface 
astrochemistry", a perfect ending for this interesting Faraday Discussion. Cornelia Jäger (Max Planck Institute for Astronomy) reviewed the first paper, entitled "Cold condensation of dust in the ISM". ${ }^{18}$ This paper shows the condensation of complex silicates with pyroxene composition at temperatures between $10-20 \mathrm{~K}$ by accretion and reaction of molecules and atoms on cold surfaces. The final condensates are efficiently formed at low temperatures and are fluffy aggregates consisting of small nanometre-sized amorphous grains. In space, gaseous precursors of these interstellar-relevant condensation processes can come from the erosion of dust by supernovae shocks. In the laboratory, the same species were formed by ablation of silicate materials with a pulsed Nd:YAG laser. Embedded in solid neon matrices, these species were further studied using spectroscopy in the UV/VIS and IR ranges. The authors note that a band at $10 \mu \mathrm{m}$ of the low temperature siliceous condensates resemble the well-known and observed $10 \mu \mathrm{m}$ feature due to interstellar silicates.

The second paper was presented by Sergio Ioppolo (California Institute of Technology) and focused on $\mathrm{THz}$ timedomain and mid-IR spectroscopy of interstellar-relevant ices at different temperatures. ${ }^{19}$ With the primary intent of providing the scientific community with an extensive $\mathrm{THz}$ ice database in support of international astronomical observations (i.e., Herschel Space Telescope, SOFIA, and ALMA), Ioppolo et al. have investigated the composition and structure of some of the most abundant interstellar ice analogues (i.e., water, carbon monoxide, and methanol). $\mathrm{THz}$ spectra of simple ices were then compared to those from more complex interstellar-relevant molecules that share the same functional groups, including formic acid and acetic acid, as well as acetaldehyde and acetone. The authors find that, since $\mathrm{THz}$ frequencies are dominated by inter- and intramolecular forces, THz spectra are sensitive to reversible and irreversible transformations within the ice, such as thermal processing of crystalline and amorphous ices, respectively.

The third and final paper before the morning break was presented by Ralf Kaiser (University of Hawaii at Manoa). Kaiser and co-workers have recently built

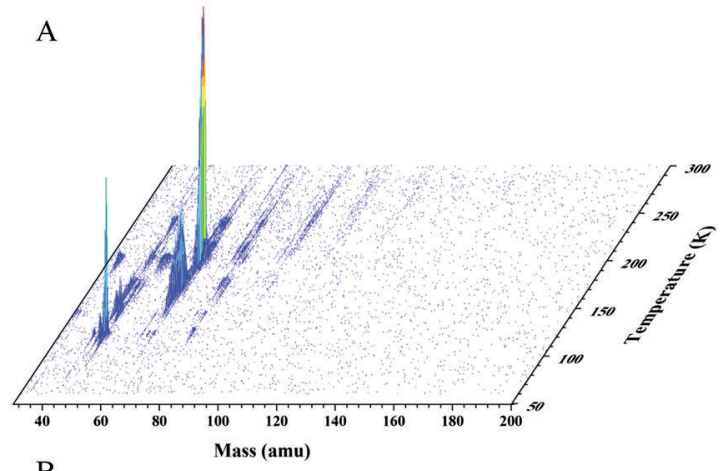

B

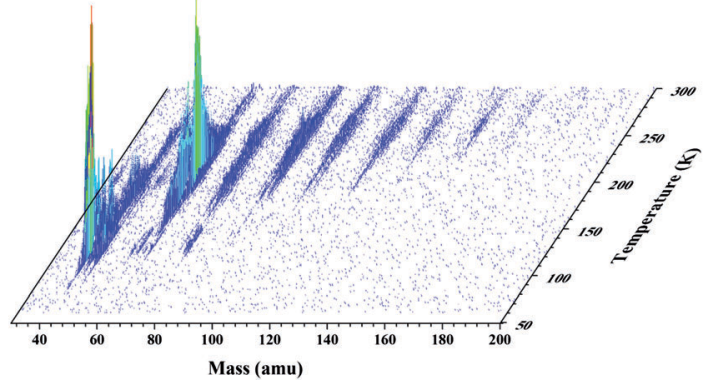

Fig. 4 Maity et al. have developed a combined infrared and time-of-flight (ToF) system to investigate molecule formation in ices triggered by ionizing radiation. $\mathrm{A}$ and $\mathrm{B}$ show the mass distribution of products formed as a function of temperature for $\mathrm{CH}_{3} \mathrm{OH}$ and $\mathrm{CH}_{3} \mathrm{OH}-\mathrm{CO}$ ices respectively. ${ }^{20}$

a new ultra-high vacuum (UHV) system to study the formation of COMs in ice at low temperatures. ${ }^{20}$ Their paper shows conclusive evidence for the formation of glycolaldehyde $\left(\mathrm{HOCH}_{2} \mathrm{CHO}\right)$ in methanol and methanol-carbon monoxide ices upon exposure to ionizing radiation at $5.5 \mathrm{~K}$ (see Fig. 4). Glycolaldehyde is the simplest monosaccharide sugar detected in numerous astrophysical environments and is also astrobiologically relevant. In this work, glycolaldehyde ice is unambiguously identified using infrared spectroscopy and results are constrained by gas-phase data acquired with a single photon-ionization reflectron time-of-flight (ReTOF) mass spectrometer. The authors discuss three possible formation reaction pathways involving the reaction of formyl radical (HCO) with a hydroxymethyl radical $\left(\mathrm{CH}_{2} \mathrm{OH}\right)$, both formed in situ via energetic processing of ices.

Gianfranco Vidali (Syracuse University, New York) opened the second part of the "New Directions" session by describing his group's recent application of modified rate equations to extracting kinetic parameters from temperature programmed desorption measurements. ${ }^{21}$ Such measurements are widely used to assess the strength of binding between molecules and grain surfaces through determination of the desorption energy. Usually, the desorption kinetics are analysed by assuming a single desorption step, that may include a distribution of adsorption energy sites, and assuming that molecules do not diffuse across the surface prior to desorption. For systems where such diffusion does occur, significant redistribution of adsorbates during the sample annealing may lead to a significant error in the derived desorption energy distribution. He et al. present a modified rate equation for thermal desorption that includes a diffusion term and demonstrate its application to several systems that exhibit desorption both with and without diffusion.

Photodesorption is a frequently invoked mechanism by which molecules can be returned to the gas phase in low temperature environments where thermal desorption is negligible. Although desorption yields have been obtained for simple molecule (e.g. $\mathrm{CO}$ ) desorption under irradiation from broadband VUV lamps, the wavelength dependence, and underlying chemical physics remains unclear. Jean-Hugues Fillion (Université Pierre et Marie Curie, Paris) outlined a series of pioneering measurements performed at 


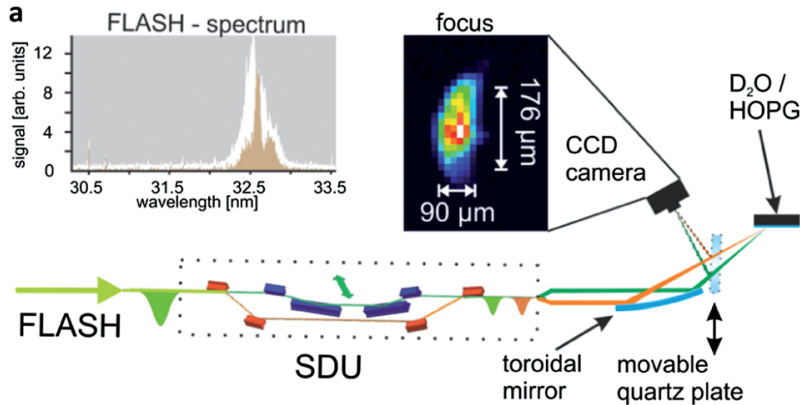

b

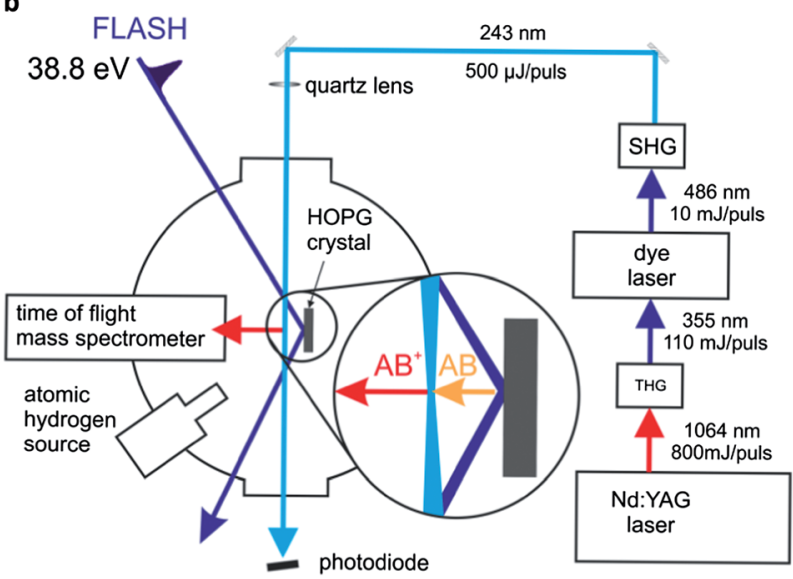

Fig. 5 Siemer et al. have used the Free Electron Laser in Hamburg (FLASH) facility to measure the ultrafast dynamics of XUV photon induced processes occurring in interstellar ices. ${ }^{23}$

the SOLEIL synchrotron. ${ }^{22}$ Fillion et al. investigate the desorption of $\mathrm{CO}$ and $\mathrm{CO}_{2}$ from $\mathrm{CO}_{2}$ ice irradiated with photons in the range 7-14 eV. Both species show desorption yields that closely follow the photon absorption cross section of $\mathrm{CO}_{2}$ above $11 \mathrm{eV}$. Pre-irradiation of $\mathrm{CO}_{2}$ produces a reservoir of $\mathrm{CO}$ molecules that can enhance $\mathrm{CO}_{2}$ desorption through an indirect mechanism involving initial photon absorption by CO molecules, extending the wavelength range over which $\mathrm{CO}_{2}$ is desorbed. Such measurements are therefore crucial to understanding nonthermal desorption in mixed interstellar ices, where indirect processes are likely to be important.

The final discussion paper was presented by Helmut Zacharias (University of Münster) who moved the discussion of photon induced ice processing to the extreme ultraviolet (XUV). In a series of unique measurements at the Free-electron laser in Hamburg (FLASH) facility, his group have used femtosecond XUV pulses to probe the photon induced reaction dynamics in simple ices $\left(\mathrm{D}_{2} \mathrm{O}, \mathrm{NO}\right)$, as well as the desorption of chemisorbed $\mathrm{H}$ atoms from graphite. ${ }^{23}$ A schematic of their experimental setup is shown in Fig. 5. The application of femtosecond pulses to investigate ultrafast dynamics provides a potentially exciting new approach for probing ice chemical processes.

The final discussion session covered some of the recent developments in astrochemical research. Prof. Kamp opened the discussion asking Dr Jäger to elaborate more on the $\mathrm{Mg}$-poor nature of their laboratory dust grains compared to the ones observed in space. Soon the discussion moved on to some detailed aspects of Cornelia Jäger's work including the high efficiency of the cold condensation process in laboratories versus space (Mr Rab), and the possibility of including $\mathrm{O}_{3}$ in the experiments to test whether olivine rather than pyroxene would be formed, as suggested by Prof. Plane. Finally, Cornelia Jäger remarked the non-equilibrium conditions of the condensation process as studied in their experiments that most likely explain the non-formation of olivine. The next series of questions from Prof. van der Tak, Dr Pontoppidan, Prof. van Dishoeck, and Prof. Linnartz were addressed to Dr Ioppolo, who showed that THz-ice features can potentially be detected in the ISM and can give information on the temperature and structure of interstellar ices. During the discussion session, Prof. Kaiser had the opportunity to reveal some of the details of their new experimental apparatus that comprises numerous in situ and gas-phase probing techniques (e.g., infrared spectroscopy, Raman spectroscopy, ultraviolet-visible spectroscopy, reflection time-of-flight mass spectrometry and photoionization). The benefits and limitations of a variety of techniques in elucidating reaction mechanisms for the formation of more complex species were discussed. As chair of the session, Prof. Linnartz introduced the new "labastro" website (www.labastro.eu) that aims to highlight the laboratory astrochemistry research being undertaken in Europe and provide information on the groups involved as well as an overview of the infrastructure available to the community at a European level. In addition, Prof. Mason mentioned the importance of database projects such as the international virtual observatory project (www.ivoa.net) and VAMDC (www. vamdc.eu). Such initiatives provide a valuable resource to the community in terms of collating data and the development of protocols for data validation and assessment. The importance of fully exploiting solid-state laboratory spectra to gain more detailed physical insights, rather than simply generating vast catalogues of spectra was also discussed. Sergio Ioppolo agreed with Prof. McCoustra's comment on the need to combine laboratory data and high-level computational calculations to understand the nature of the collective modes of the condensed phase. Prof. Dulieu asked Prof. Zacharias and Prof. Fillion if they had any insights as to the sharing of the incident photon energy during photo-induced desorption processes, as well as the exact role of the substrate. Prof. Fillion explained that vibrational relaxation within the ice matrix appears to compete with the desorption channel and that the nature of the substrate has a strong effect on quenching of photodesorption, resulting from differences in adsorbate-substrate binding. Prof. Zacharias agreed, highlighting the difference in 
mechanisms between single ice layers, where the underlying substrate plays a central role in the energy transfer process, and thick ice layers where excitation of vibrational motion within the ice lattice becomes important.

The challenge of summarizing the papers discussed during the meeting fell to Eric Herbst (University of Virginia) who provided an entertaining overview of the research presented. In addition to tying together the various strands of astrochemistry, he also reminded the attendees of the continuing advances in gas-phase astrochemistry that were reported in several interesting posters, despite not being as well represented through the papers. To conclude, he set the scene for the future, looking forward to further discussion meetings. Observational astrochemistry is entering a new era with the ongoing construction of ALMA and the launch of JWST in 2019. It is clear that astrochemists across the board will be kept busy in the years to come, providing plenty of substance for future discussions.

To round off the meeting, Martin McCoustra (Heriot-Watt University, Edinburgh) thanked the local organisers from Leiden and the staff of the RSC for hosting and organizing such a fascinating and stimulating discussion meeting before inviting the attendees to meet for a final lunch together before departure. The Faraday Discussion 168 volume including all of the general discussions is published by the Royal Society of Chemistry.

\section{Acknowledgements}

J.D.T. acknowledges support from the University of Münster. S.I. acknowledges support from a Niels Stensen Fellowship and a Marie Curie Fellowship (FP7-PEOPLE2011-IOF-300957). C.W. acknowledges support from the Netherlands Organisation for Scientific Research (NWO, program number 639.041.335).

\section{References}

1 K. M. Pontoppidan and S. Blevins, Faraday Discuss., 2014, 168, 49.

2 K. I. Öberg, E. C. Fayolle, J. B. Reiter and C. Cyganowski, Faraday Discuss., 2014, 168, 81.

3 V. V. Guzman, J. Pety, P. Gratier, J. R. Goicoechea, M. Gerin, E. Roueff, F. Le Petit and J. Le Bourlot, Faraday Discuss., 2014, 168, 103.

4 E. Bergin, L. I. Cleeves, N. Crockett and G. Blake, Faraday Discuss., 2014, 168, 61.

5 E. Congiu, M. Minissale, S. Baouche, H. Chaabouni, A. Moudens, S. Cazaux G. Manico, V. Pirronello and F. Dulieu, Faraday Discuss., 2014, 168, 151.

6 H. J. Kimber, C. P. Ennis and S. D. Price, Faraday Discuss., 2014, 168, 167.

7 Y. Oba, K. Osaka, N. Watanabe, T. Chigai and A. Kouchi, Faraday Discuss., 2014, $168,185$.
8 M. W. Lee and M. Meuwly, Faraday Discuss., 2014, 168, 205.

9 A. L. Skov, J. D. Thrower and L. Hornekær, Faraday Discuss., 2014, 168, 223.

10 N. J. Mason, B. Nair, S. Jheeta and E. Szymanska, Faraday Discuss., 2014, 168, 235.

11 M. D. Boamah, K. K. Sullivan, K. E. Shulenberger, C. M. Soe, L. M. Jacob, F. C. Yhee, K. E. Atkinson, M. C. Boyer, D. R. Haines and C. R. Arumainayagam, Faraday Discuss., 2014, 168, 249.

12 B. Maté, I. Tanarro, M. A. Moreno, M. JiménezRodondo, R. Escribano and C. J. Herrero, Faraday Discuss., 2014, 168, 267.

13 A. P. Jones, N. Ysard, M. Köhler, L. Fanciullo, M. Bocchio, E. Micelotta, L. Verstraete and V. Guillet, Faraday Discuss., 2014, 168, 313.

14 T. Lamberts, X. de Vries and H. M. Cuppen, Faraday Discuss., 2014, 168, 327.

15 B. Parise, P. Bergman and K. Menten, Faraday Discuss., 2014, 168, 349.

16 J. M. C. Rawlings, D. A. Williams, S. Vita, C. Cecchi-Pestellini and W. W. Duley, Faraday Discuss., 2014, 168, 369.

17 C. Walsh, E. Herbst, H. Nomura, T. J. Millar and S. Widicus Weaver, Faraday Discuss., 2014, 168, 389.

18 G. Rouillé, C. Jäger, S. A. Krasnokutski, M. Krebsz and T. Henning, Faraday Discuss., 2014, 168, 449.

19 S. Ioppolo, B. A. McGuire, M. A. Allodi and G. A. Blake, Faraday Discuss., 2014, 168, 461.

20 S. Maity, R. I. Kaiser and B. M. Jones, Faraday Discuss., 2014, 168, 485.

21 J. He and G. Vidali, Faraday Discuss., 2014, $168,517$.

22 J.-H. Fillion, E. C. Fayolle, X. Michaut, M. Doronin, L. Philippe, J. Rakovsky, C. Romanzin, N. Champion, K. I. Öberg, H. Linnartz and M. Bertin, Faraday Discuss., 2014, 168, 533.

23 B. Siemer, S. Roling, R. Frigge, T. Hoger, R. Mitzner and H. Zacharias, Faraday Discuss., 2014, 168, 553. 